\title{
The population of Near Earth Asteroids in coorbital motion with Venus
}

\author{
M.H.M. Morais ${ }^{\mathrm{a}, *}$, A. Morbidelli ${ }^{\mathrm{b}}$ \\ a Grupo de Astrofísica da Universidade de Coimbra, Observatório Astronómico de Coimbra, Santa Clara, 3040 Coimbra, Portugal \\ b Observatoire de la Côte d'Azur, BP 4229, Boulevard de l'Observatoire, Nice Cedex 4, France
}

Received 13 January 2006; revised 10 April 2006

Available online 14 August 2006

\begin{abstract}
We estimate the size and orbital distributions of Near Earth Asteroids (NEAs) that are expected to be in the 1:1 mean motion resonance with Venus in a steady state scenario. We predict that the number of such objects with absolute magnitudes $H<18$ and $H<22$ is $0.14 \pm 0.03$ and $3.5 \pm 0.7$, respectively. We also map the distribution in the sky of these Venus coorbital NEAs and we see that these objects, as the Earth coorbital NEAs studied in a previous paper, are more likely to be found by NEAs search programs that do not simply observe around opposition and that scan large areas of the sky.

(c) 2006 Elsevier Inc. All rights reserved.
\end{abstract}

Keywords: Asteroids, dynamics; Resonances

\section{Introduction}

In Morais and Morbidelli (2002), hereafter referred to as Paper I, we estimated the population of Near Earth Asteroids (NEAs) that are in the 1:1 mean motion resonance (i.e., are coorbital $^{1}$ ) with the Earth in a steady state scenario where NEAs are constantly being supplied by the main belt sources (Morbidelli et al., 2002). Here, we will apply the same methodology to estimate the population of NEAs that are in the 1:1 mean motion resonance with Venus.

Numerical integrations of the orbits of NEAs showed that these objects can become temporary coorbitals of the terrestrial planets. Wiegert and Innanen (1997) showed that (3753) Cruithne has an asymmetric horseshoe orbit ${ }^{2}$ with the Earth. Namouni (1999) later explained in detail the behavior of this

\footnotetext{
* Corresponding author. Rua Padre Himalaia 94, $1^{\circ}$ Dt. Frt. 4100-553 Porto, Portugal.

E-mail addresses: hmorais@mat.uc.pt (M.H.M. Morais), morby@obs-nice.fr (A. Morbidelli).

1 Coorbital objects can have tadpole orbits around the triangular equilibria $L_{4}$ or $L_{5}$, horseshoe orbits, quasi-satellite orbits, compound and transition orbits that include the previous modes (Namouni, 1999; Nesvorný et al., 2002).

2 Asymmetric horseshoe (tadpole) orbits are compound orbits that involve horseshoe (tadpole) modes and quasi-satellite modes (Namouni, 1999; Nesvorný et al., 2002).
}

object with a theory based on the restricted three body problem at high eccentricity and inclination. Christou (2000) performed a $0.2 \mathrm{Myr}$ integration of the orbits of NEAs in the vicinity of the terrestrial planets, namely (3362) Khufu, (10563) Izhdubar, 1994 TF2 and 1989 VA, showing that the first three could become coorbitals of the Earth while the fourth could become coorbital of Venus. Michel (1997) had previously performed a 2 Myr integration of the orbit of (45660) Nereus showing that this object could be captured in the 1:1 mean motion resonance with Venus.

Recently, other examples of current Earth and Venus coorbitals were found. Connors et al. (2002) showed that 2002 AA29 is in a horseshoe orbit with the Earth. Wiegert et al. (2002) showed that 2000 PH5 and 2001 GO2 are also in horseshoe orbits with the Earth. Connors et al. (2004) showed that 2003 YN107 is a quasi-satellite of the Earth. Finally, we know 2 examples of current Venus coorbitals: Mikkola et al. (2004) showed that 2002 VE68 is a quasi-satellite of Venus and Brasser et al. (2004) showed that 2001 CK32 has an asymmetric horseshoe orbit with Venus.

In this paper we will test the hypothesis of the existence of a population of Venus coorbital NEAs which are constantly being supplied by main belt sources. Using as a starting point the predictions of the NEA model of Bottke et al. (2000, 2002), we will estimate the steady state number (according to size) of 
Venus coorbital NEAs, we will obtain their orbital elements' distribution, and finally we will investigate their observability. We will also compare these results with those obtained in Paper I for the population of Earth coorbital NEAs.

\section{Methodology}

The methodology for estimating the population of Venus coorbital NEAs is the same as the one described in Paper I. We followed the evolution of test bodies with $e$ and $I$ chosen according to the NEA model of Bottke et al. (2000, 2002) for $0.8<a<0.9 \mathrm{AU}$ (our intermediate source region) as they became coorbital with the Venus (our target region) and until they collide with the Sun or a terrestrial planet, or achieve $a>10 \mathrm{AU}$. The orbital elements of these test bodies (as well as those of the planets) were outputted every 100 years as a compromise between being able to accurately identify coorbital motion with Venus (the coorbital period of Venus' tadpole orbits is about 150 years $^{3}$ ) and generating manageable amounts of data.

In order to decide if a test body is captured in the 1:1 mean motion resonance with Venus we checked for oscillation of its semi-major axis around $0.72 \mathrm{AU}$. Oscillations with periodicity larger than 10,000 years (thus much larger than a typical coorbital period; see above) or that happened for less than 10 consecutive output times were rejected.

As in Paper I, we stress that the setting of the intermediate source (IS) as the NEA region with $0.8<a<0.9 \mathrm{AU}$ is correct as any test bodies coming from the main belt will enter this region before becoming Venus coorbitals and are also likely to remain in this region for a time larger than 10,000 years (the interval at which the output of the numerical integrations is sampled in the NEA model) with the exception of those rare cases in which they jump over the IS region due to a deep planetary close encounter. The advantage of this choice of IS region is that, in this way, we increase the likelihood of a test body being captured as a Venus coorbital (because we start with a large population in a region already quite close to the target region) and can therefore obtain better statistics than if we followed the test bodies all the way from the main belt sources. Obviously, we could not follow this methodology if we did not have yet a prediction of the NEAs orbital and size distribution in the region with $0.8<a<0.9$ AU.

In a steady state scenario, the population in the target (TR) region is

$N_{\mathrm{TR}}=F \times L_{\mathrm{TR}}$,

where $F$ is the flux of entrance in the TR region and $L_{\mathrm{TR}}$ is the mean life-time in the TR region.

The quantity $L_{\mathrm{TR}}$ can be obtained directly from the simulations. In order to compute $F$, we follow the same method described in Paper I. We know that our simulated situation corresponds to a steady state scenario in which we suddenly stop

\footnotetext{
3 Coorbital period is the period of the motion of the coorbital object relative to the planet. Tadpole orbits have coorbital period equal to $[(27 / 4) \mu]^{-1 / 2}$ orbital periods (Message, 1966) where $\mu$ is the planet to Sun mass ratio.
}

feeding the IS region. Therefore, the subpopulation of the IS region that feeds the TR region, $N_{s}$, which is initially a fraction $f$ of the total population of the IS region (i.e., $N_{s}(0)=f N_{\text {IS }}$ ), starts decaying into the TR region at a rate

$\frac{\mathrm{d} N_{s}}{\mathrm{~d} t}=-r_{\mathrm{IS}} N_{s}$,

where $r_{\mathrm{IS}}$ is the fractional decay rate into the TR region which is approximately constant for small $t$.

On the other hand, the number of bodies in the IS region that still have not entered the TR region at time $t$ is

$N(t)=N_{S}(t)+N_{\mathrm{IS}}-f N_{\mathrm{IS}}$,

where $N_{\mathrm{IS}}$ is the initial population in the IS region.

We obtain $N_{s}(t)$ by solving Eq. (2) with initial condition $N_{s}(0)=f N_{\text {IS }}$. Then we substitute this into Eq. (3) and expand it in a Taylor series up to first order around $t=0$, obtaining

$N(t)=N_{\text {IS }} \exp \left[-r_{\text {IS }} f t\right]$,

which is valid for small $t$.

Finally, as the chosen initial conditions are representative of the steady state orbital distribution in the IS region, the flux of entrance in the TR region is given by

$F=r_{\text {IS }} f N_{\text {IS }}$

and we can estimate $r_{\mathrm{IS}} f$ by fitting the data from the simulations to Eq. (4).

\section{Results and discussion}

\subsection{The numerical integration scheme}

As in Paper I, all our numerical integrations were made using the "swift-rmvs3" integrator (Levison and Duncan, 1994) with a time-step of 4 days. This is a modification of the symplectic algorithm proposed by Wisdom and Holman (1991) which is able to deal with planetary close encounters.

\subsection{Statistics from the numerical integrations}

We followed a set of 2000 test bodies initially in the IS region as their orbits evolve subject to gravitational perturbations from the 7 planets Venus to Neptune. This set was followed first for $5 \mathrm{Myr}$, in order to estimate the flux of entrance in the TR region.

Now, according to Eq. (5), the flux of entrance in the TR region depends on the rate $r_{\text {IS }} f$ and $N_{\text {IS }}$ (the steady state number of NEAs in the IS region).

The quantity $r_{\mathrm{IS}} f$ is, according to Eq. (4), the absolute value of the slope of $\ln [N(t)]$. In Fig. 1a we show the plot of $\ln [N(t)]$ and in Fig. 1b we show the plot of $r_{\mathrm{IS}} f$; this latter one is the absolute value of the slope of $\ln [N(t)]$ obtained by applying the method of linear regression in the interval $[0, T]$ where $T$ ranges from 0.5 to $5 \mathrm{Myr}$.

Now, the computation of the fractional decay rate for $T$ too small is unreliable due to the small number of data points available. On the other hand, for $T$ too large we are attempting to fit 

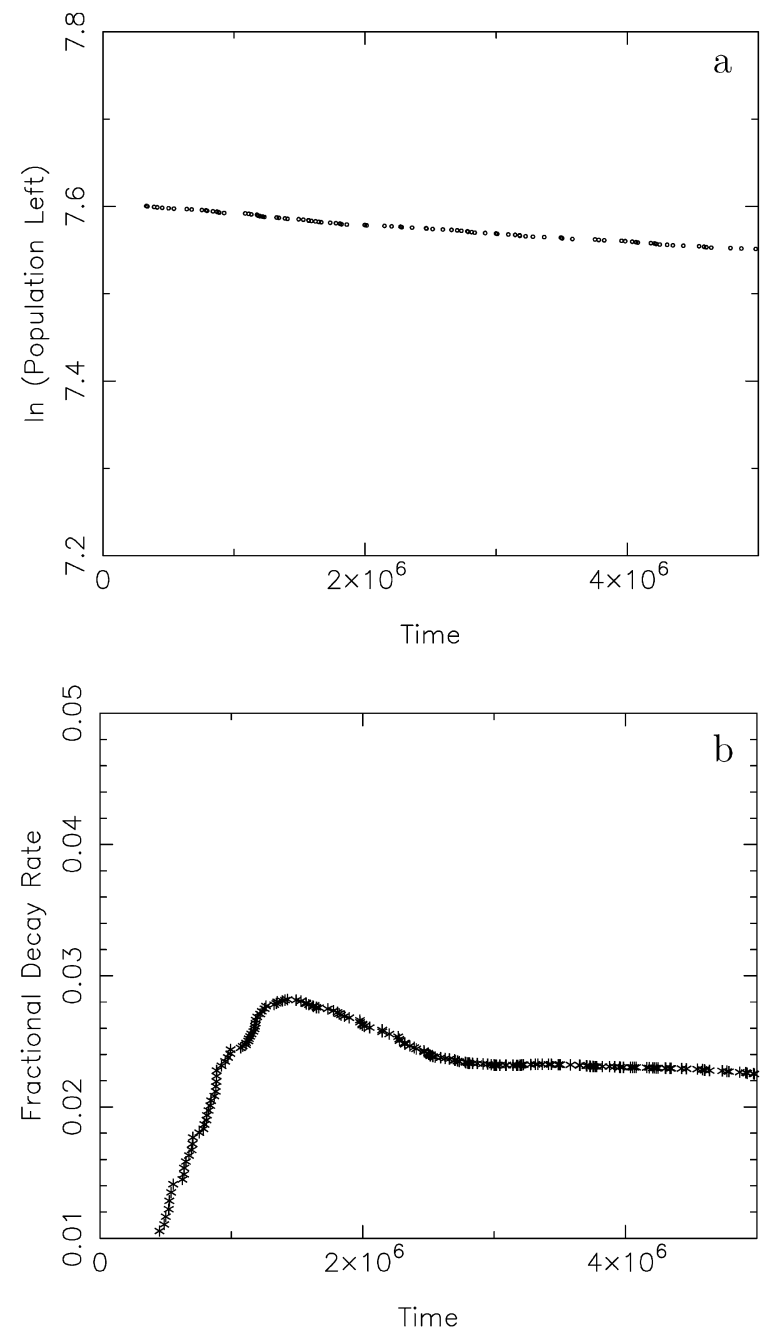

Fig. 1. The decay rate from the IS region into the TR region: (a) logarithm of the population left in the IS region as a function of time; (b) fractional decay rate into the TR region obtained by fitting a straight line to the graphic (a) in the interval $[0, T]$.

a straight line to a part of $\ln [N(t)]$ where the decay rate deviates from an exponential law. By inspection of Fig. 1b we decided to compute an average fractional decay rate by including only the values of the slopes between $T=1$ and $3 \mathrm{Myr}$, which gives $r_{\text {IS }} f=-0.0255 \pm 0.0015 \mathrm{Myr}^{-1}$.

Finally, from the NEA model (Bottke et al., 2000, 2002) we can obtain the steady state number of NEAs with absolute magnitude $H<H_{0}$ in the IS region. In particular, $N_{\text {IS }}=$ $16.2 \pm 2.4$ for $H_{0}=18$ and this value scales approximately as $10^{0.35\left(H_{0}-18\right)}$ for $18<H_{0}<22$. We can then apply Eq. (5) to obtain the flux of entrance in the TR region: this is $F=$ $0.41 \pm 0.09$ test bodies $\mathrm{Myr}^{-1}$ for $H<18$.

Now, according to Eq. (1), in order to obtain the steady state number of Venus coorbital NEAs we need to compute $L_{\mathrm{TR}}$ (the mean life-time in the target region). In order to obtain this quantity, we followed up to 100 Myr the set of test bodies that after $5 \mathrm{Myr}$ had at some stage been coorbital with Venus. This integration timespan proved to be long enough for our purposes as, at the end of it, not only there were very few surviving test bodies but also new captures in the 1:1 mean motion resonance were then very unlikely (indeed at the end of the $100 \mathrm{Myr}$ timespan the cumulative time spent in the TR region had almost reached a plateau). We obtained a mean life-time in the 1:1 mean motion resonance with Venus, $L_{\mathrm{TR}}=0.34 \mathrm{Myr}$. Applying Eq. (1) we can then predict the steady state number of Venus coorbital NEAs: this is $N_{\mathrm{TR}}=0.14 \pm 0.03$ and $N_{\mathrm{TR}}=3.5 \pm 0.7$ for $H<18$ and $H<22$, respectively. ${ }^{4}$

In Paper I, we saw that the steady state number of Earth coorbital NEAs was $N_{\mathrm{TR}}=0.65 \pm 0.12$ and $N_{\mathrm{TR}}=16 \pm 3$ for $H<18$ and $H<22$, respectively. Therefore, the population of Venus coorbitals is a factor $0.21 \pm 0.09$ of the population of Earth coorbitals. Since the average lifetimes in the 1:1 mean motion resonance are very similar, this difference is due to the fact that the flux of coorbital captures is much smaller for Venus than for the Earth.

We can understand why this is so. The flux of coorbital captures is given by the number of bodies, $N_{\mathrm{IS}}$, available in the IS region, times the capture probability. We can obtain a rough measure of the latter quantity by assuming that it scales as the area of the coorbital region ${ }^{5}$ which is $4 \pi a^{2}(\mu / 3)^{1 / 3}$, where $a$ is the planet's semi-major axis and $\mu$ is the planet to Sun mass ratio. Therefore, the ratio of the capture probabilities for Venus and the Earth is 0.486 . On the other hand, the ratio of the number of bodies available in Venus' and Earth's IS regions is 0.516; hence the ratio of the coorbital capture fluxes for Venus and the Earth is about 0.25. This agrees, within the error bars, with the value $0.21 \pm 0.09$ which we obtained from the numerical integrations.

We also saw that these Venus' coorbitals, like the Earth coorbitals, typically experience several captures/escapes in/from the 1:1 mean motion resonance. The average duration of a Venus coorbital episode is around 32,000 years with 8 episodes lasting longer than 1 Myr. In Paper I, we saw that the average duration of an Earth coorbital episode was around 25,000 years with no episodes lasting longer than 1 Myr. The existence of long coorbital episodes for Venus as compared to the Earth can be explained by the respective eccentricity distributions, as we will see in Section 3.4 .

\subsection{Comparison between model and observations}

We currently know two Venus coorbitals: these are 2001 CK32 with $H=18.9$ and 2002 VE68 with $H=20.3$. On the other hand, we currently know five Earth coorbitals. However, with the exception of (3753) Cruithne with $H=15.1$, all the other objects are quite small with $H>22$. Our work is based on the model by Bottke et al. $(2000,2002)$ which only applies in the size range corresponding to $H<22$.

In Table 1 we show the predicted number of Venus coorbital NEAs (Section 3.2) and Earth coorbital NEAs (Paper I, Section 3.2) with $H<H_{0}$ for $18 \leqslant H_{0} \leqslant 22$. At first sight we could

\footnotetext{
4 Note that the relative error in $N_{\mathrm{TR}}(21 \%)$ is simply the sum of the relative error in the slope $r_{\mathrm{IS}} f(6 \%)$ with the relative error in $N_{\mathrm{IS}}(15 \%)$.

5 The coorbital region can be defined as the area of the circular ring with mean radius $a$ (the planet's semi-major axis) and half width $a(\mu / 3)^{1 / 3}$ (the planet's Hill's radius).
} 
Table 1

Estimated number of Venus and Earth coorbital NEAs

\begin{tabular}{lllllr}
\hline Coorbitals & $H<18$ & $H<19$ & $H<20$ & $H<21$ & \multicolumn{1}{c}{$H<22$} \\
\hline Venus & $0.14 \pm 0.03$ & $0.31 \pm 0.07$ & $0.70 \pm 0.15$ & $1.57 \pm 0.34$ & $3.5 \pm 0.7$ \\
Earth & $0.65 \pm 0.12$ & $1.45 \pm 0.27$ & $3.26 \pm 0.60$ & $7.29 \pm 1.35$ & $16.3 \pm 3.0$
\end{tabular}

conclude that the survey for Venus coorbitals is complete for $H<21$ (since we know 2 objects) while the survey for Earth coorbitals is complete for $H<18$ (since we know one object). However, as already stated in Paper I, the quantities in Table 1 are average estimates of the population of Venus' and Earth's coorbital NEAs and thus should be compared with the number of NEAs which become coorbital with these planets over a large enough timespan.

Christou (2000) searched a database of known NEAs for objects with semi-major axis close to that of Venus and the Earth, and followed them for $0.2 \mathrm{Myr}$. He found that 1989 VA $(H=17.9)$ had a $3 / 7$ probability of becoming coorbital with Venus during the integration timespan, while (10563) Izhdubar $(H=16.9)$, (3362) Khufu $(H=18.3)$ and 1994 TF2 $(H=19.3)$ had each $4 / 7$ probability of becoming coorbital with the Earth over the same timespan (Christou, 2005, private communication). Additionally, we know that (3753) Cruithne ( $H=15.1)$ is currently a coorbital of the Earth. We can thus say that, at the time of Christou (2000) article, we knew 0.43 objects with $H<18$ that became coorbital with Venus and 2.14 objects with $H \lesssim 18$ (or, excluding (3362) Khufu, 1.57 objects with $H<18$ ) that became coorbital with the Earth during the $0.2 \mathrm{Myr}$ timespan.

On the other hand, according to our model, the number of objects with $H<H_{0}$ that become coorbital with a planet over a timespan $\Delta t$ is $N \times \Delta t / \Delta t_{1: 1}$, where $N$ is the steady state number of coorbitals and $\Delta t_{1: 1}$ is the average duration of a coorbital episode.

In the case of Venus we have $\Delta t_{1: 1}=32,000$ years hence we estimate that $(0.14 \pm 0.03) \times \Delta t / \Delta t_{1: 1}=0.88 \pm 0.19$ objects with $H<18$ become coorbital with this planet during the $0.2 \mathrm{Myr}$ timespan. We can thus say that, at the time of the search by Christou (2000), the completeness of observations for $H<18$ near $a=0.72$ AU was 39-59\% The NEA model presented in the article of Bottke et al. (2000), written at around the same time, predicts completeness of $37-47 \%$ for $H<18$ near $a=0.72 \mathrm{AU}$. We can thus conclude that our estimates for the population of Venus coorbitals are in reasonable agreement with the observations.

In the case of the Earth we have $\Delta t_{1: 1}=25,000$ years (see Paper I) hence we estimate that $(0.65 \pm 0.12) \times \Delta t / \Delta t_{1: 1}=$ $5.2 \pm 0.9$ objects with $H<18$ become coorbital with this planet during the $0.2 \mathrm{Myr}$ timespan. We can thus conclude that at the time of the search by Christou (2000), the completeness of observations for $H \lesssim 18$ near $a=1$ AU was $35-49 \%$. However, if we exclude (3362) Khufu with $H=18.3$ from the sample, we see that the completeness of observations for $H<18$ near $a=1$ AU was 26-36\%. The NEA model of Bottke et al. (2000) predicts completeness of $31-40 \%$ for $H<18$ near $a=0.72 \mathrm{AU}$. Therefore, as stated in Paper I, we conclude that
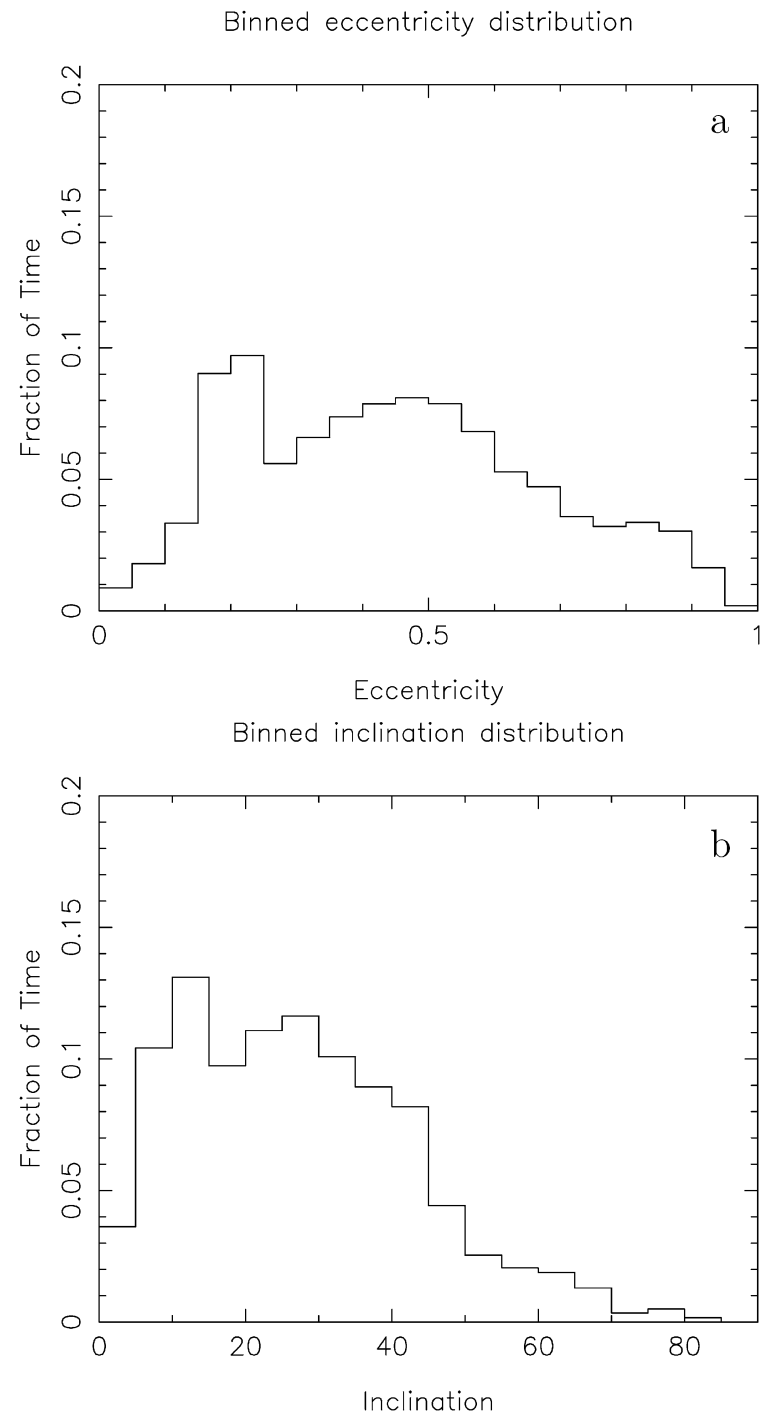

Fig. 2. The binned eccentricity (a) and inclination (b) distributions for the Venus coorbitals from our simulations.

our estimates for the population of Earth coorbitals are in good agreement with the observations.

\subsection{Eccentricity and inclination distributions}

The binned eccentricity and inclination distributions, obtained while the test bodies were in the 1:1 mean motion resonance with Venus, are shown in Figs. 2a and 2b, respectively. We see that the distribution of eccentricities is broad. There is a significant peak centered at $e=0.2$; this is due to a long coorbital capture (as an $L_{4}$ trojan) that last about $9.5 \mathrm{Myr}$ which we show in Fig. 3. Such long coorbital capture represents about $11 \%$ of the total time spent in the 1:1 mean motion resonance by all test particles in the simulation. In Fig. 3 we see that the average eccentricity is around 0.2 , always remaining below 0.3 . As Venus coorbitals require eccentricity $e \geqslant 0.388$ in order to be on Earth crossing orbits, this object is protected against close encounters with the Earth therefore its orbit is very stable.

From the eccentricity distribution we can compute the fraction of time spent with $e \geqslant 0.388$ which is $56 \%$. This gives 

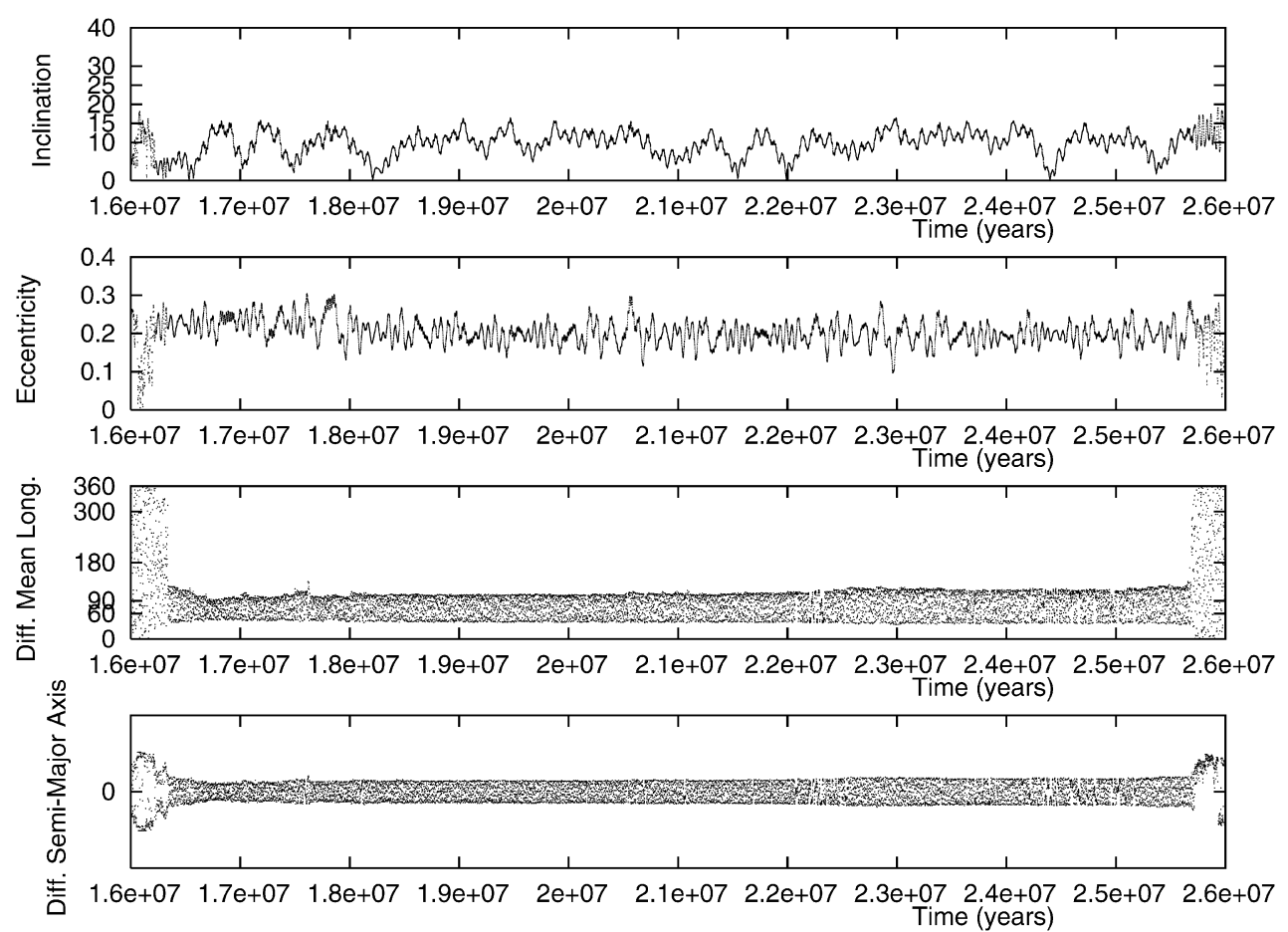

Fig. 3. Long Venus' coorbital episode ( $L_{4}$ trojan). From bottom to top panel: difference in semi-major axes between the coorbital and Venus, difference in mean longitudes between the coorbital and Venus, eccentricity and inclination of coorbital.

us a measure of the probability of a Venus coorbital having an Earth-crossing orbit. Conversely, a Venus coorbital has a $44 \%$ probability of not having an Earth-crossing orbit which lead us to conclude that $44 \%$ of these objects are IEOs (Inner Earth Objects). However, as the eccentricity distribution in Fig. 2a is not smooth, we may be overestimating the percentage of IEOs within the population of Venus coorbitals possibly by as much as $10 \%$.

On the other hand, in Paper I we saw that most Earth coorbitals had Venus crossing orbits and that many had also Mars crossing orbits. We can also quantify these probabilities by computing the fraction of time spent with $e \geqslant 0.28$ and $e \geqslant 0.52$ from the respective eccentricity distribution. This gives $75 \%$ and 35\% for Venus' and Mars' crossing orbits, respectively. Therefore, an Earth coorbital has only 25\% probability of not having a (potentially unstable) Venus crossing orbit which explains why the coorbital episodes in the Earth simulations tend to be shorter than those in the Venus simulations.

The inclination distribution in Fig. $2 b$ allows us to estimate the most likely inclinations for Venus coorbitals; the probability for orbits with $5^{\circ}<I<40^{\circ}$ is $75 \%$. In Paper I we saw that most Earth coorbitals had $10^{\circ}<I<45^{\circ}$; the associated probability is $74 \%$. We thus conclude that the average inclination of Venus coorbitals is only slightly smaller (about $5^{\circ}$ ) than the average inclination of Earth coorbitals.

\subsection{The distribution of coorbitals in the sky}

We obtain the distribution in the sky of Venus coorbitals following the same method we used in Paper I. We compute the time spent by the Venus coorbitals in each $5^{\circ} \times 5^{\circ}$ cell in eclip- tic longitude and latitude coordinates; then we normalize this by the total time spent in the 1:1 mean motion resonance thus obtaining the residence-time probability distribution of Venus coorbital NEAs.

In Fig. 4a we show the average probability density of Venus coorbital NEAs as function of geocentric ecliptic longitude and latitude. As Venus is not fixed in this coordinate frame, this is the residence-time probability distribution that we expect to see if we observe the sky over a time which is longer than the period of motion of a Venus' coorbital relative to the Earth (if the coorbital exactly shares Venus' orbit this is about 1.6 years). Such scenario makes sense in the context of the NEAs search programmes that have been scanning the sky for several years (http://neo.jpl.nasa.gov/programs).

In Fig. 4b we show the average extinction, $V-H$, i.e., the difference between the visual magnitude, $V$, and the absolute magnitude, $H$. This was obtained by computing the average of the individual extinctions (according to Eq. (7) from Paper I with the same slope parameter used there) for each output timestep spent by a coorbital test body within each $5^{\circ} \times 5^{\circ}$ cell in geocentric ecliptic longitude and latitude coordinates.

In Fig. $4 \mathrm{c}$ we show the average rate of longitudinal motion and in Fig. 4d we show the average rate of latitudinal motion, both with respect to the Earth and as functions of geocentric ecliptic coordinates. We would like to point out a mistake in our previous article: in Figs. 4c and 4d (Paper I) we do not see the rates of motion but the relative velocities with respect to the Earth in units $4.25 \mathrm{AU} /$ century. The correct figures with the rates of motion of Earth coorbitals in geocentric ecliptic coordinates can be found in http://www.astro.mat.uc.pt/ hmorais/ earthcoorbs.pdf. 

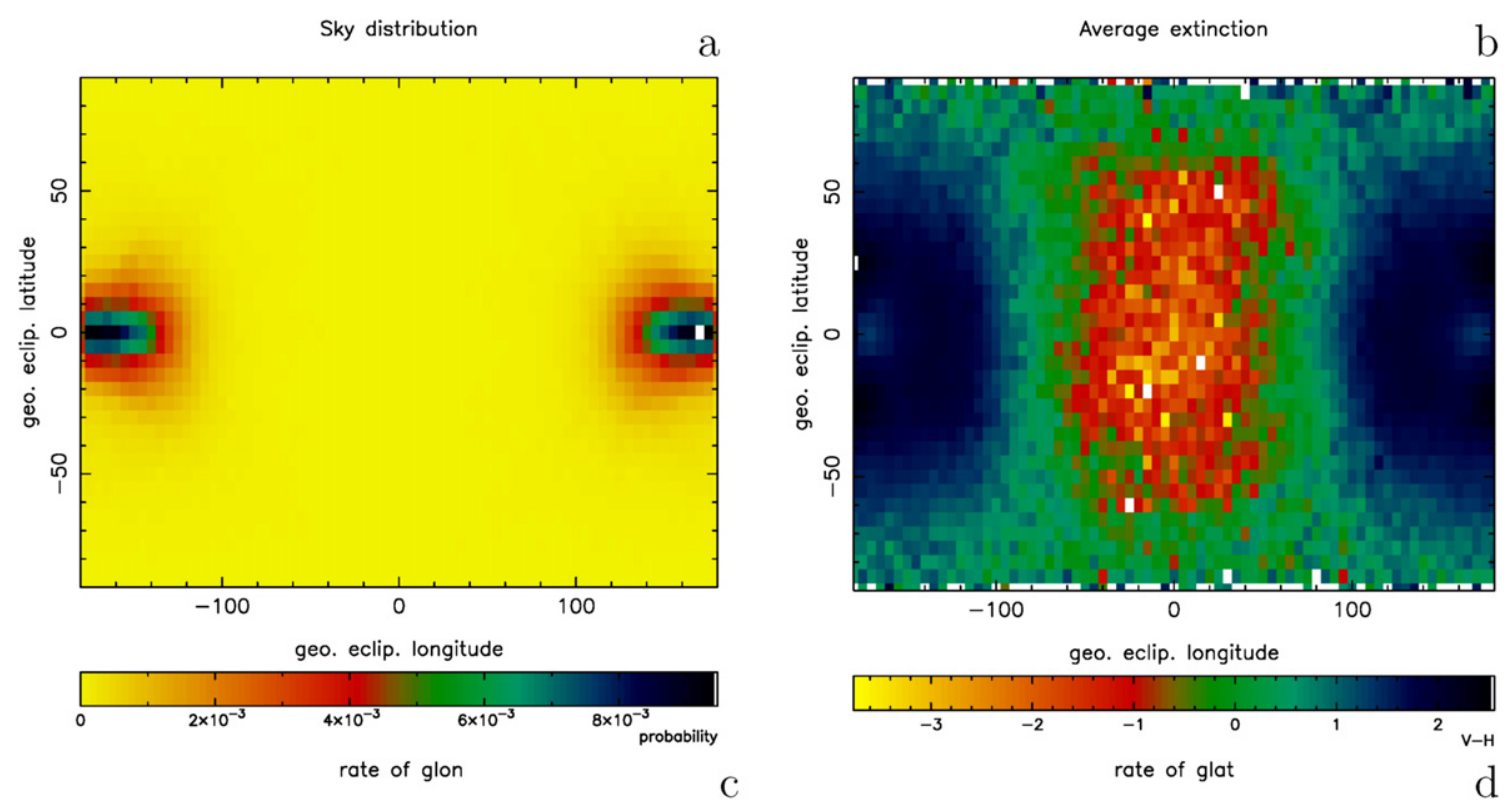

$\mathrm{b}$
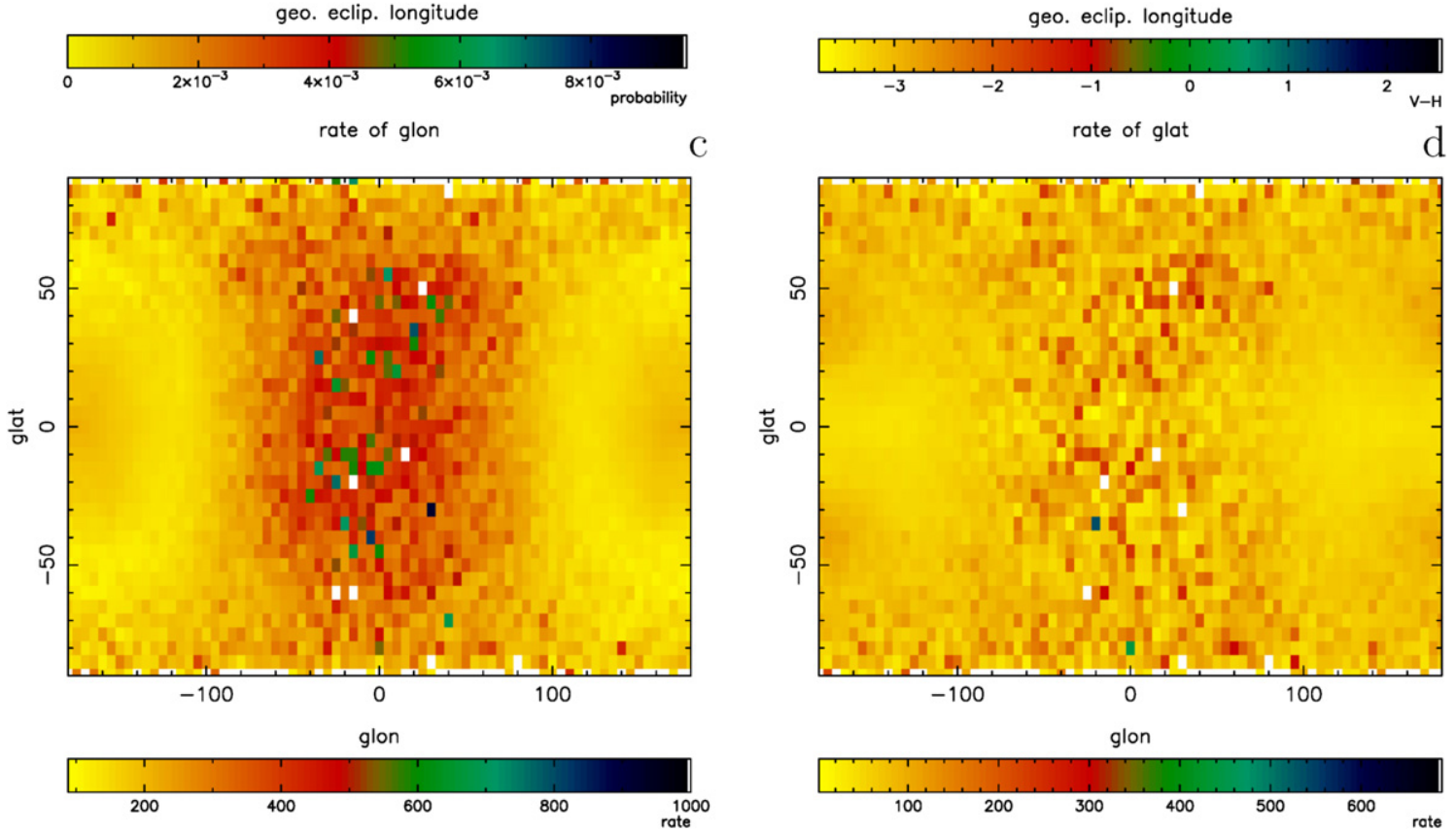

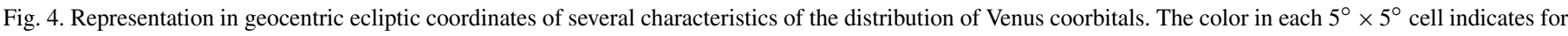

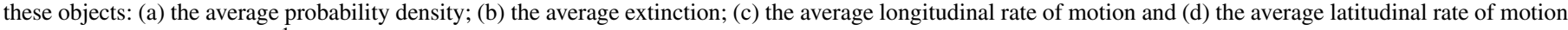
(both rates measured in ${ }^{\prime \prime} \mathrm{h}^{-1}$ ). The opposition has geocentric ecliptic latitude and longitude $0^{\circ}$.

The probability density of Venus coorbitals in geocentric ecliptic coordinates, at a given moment of time, will depend on the position of Venus relative to the Earth. We show some examples in Fig. 5a (Venus at inferior conjunction with the Earth), Fig. 5b (Venus at superior conjunction with the Earth), Fig. 5c (Venus at $90^{\circ}$ from the Earth) and Fig. $5 \mathrm{~d}$ (Venus at $-90^{\circ}$ from the Earth). In Fig. 6 we show the geocentric ecliptic longitude with respect to opposition, glon, of Venus and the Lagrangian points $L_{3}, L_{4}$ and $L_{5}$, as a function of the position of Venus relative to the Earth which is measured by the Venus-Sun-Earth angle.

Although the distributions in Figs. 5a and $5 \mathrm{~b}$ are not symmetric, these asymmetries are solely due to the long coorbital capture around $L_{4}$ shown in Fig. 3 (in fact, while in Fig. 5a $L_{4}$ is located at glon $\approx 136^{\circ}$ and $L_{5}$ at glon $\approx-136^{\circ}$, in Fig. $5 \mathrm{~b}$ $L_{4}$ is located at glon $\approx-155.5^{\circ}$ and $L_{5}$ at glon $\approx 155.5^{\circ}$ ) and therefore do not imply "real" asymmetries in the expected distribution of Venus coorbitals. On the other hand, the asymmetries in the distributions in Figs. $5 \mathrm{c}$ and $5 \mathrm{~d}$ are due to a "real" asymmetry in the location of Venus' Lagrangian points in the geocentric frame for these configurations, as can be seen in Fig. 6.

The small asymmetries in Figs. 5c, 5d and 6, which are function of the location of Venus when viewed from the Earth, do not change the overall properties of the probability density. Moreover, as can be seen in Fig. 4a, these small asymmetries average out with the motion of Venus with respect to the Earth. We will use this average probability density in order to make predictions about the distribution in geocentric ecliptic coordinates of Venus coorbitals. We recall that, as stated above, this average distribution makes more sense in the context of longterm surveys like the NEAs search programmes.

By looking at Fig. 4a above and Fig. 4a from Paper I we see that both Venus' and Earth's coorbital NEAs when viewed 
Sky distribution

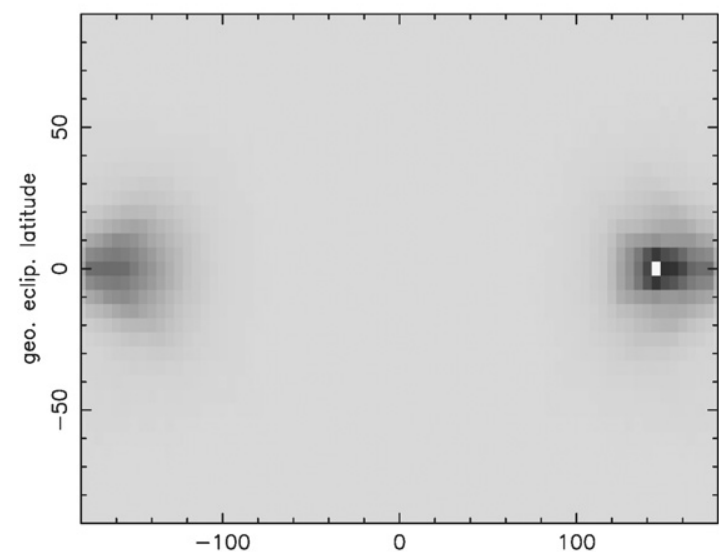

geo. eclip. Iongitude

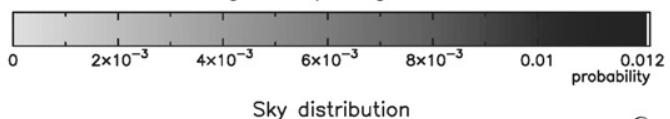

Sky distribution

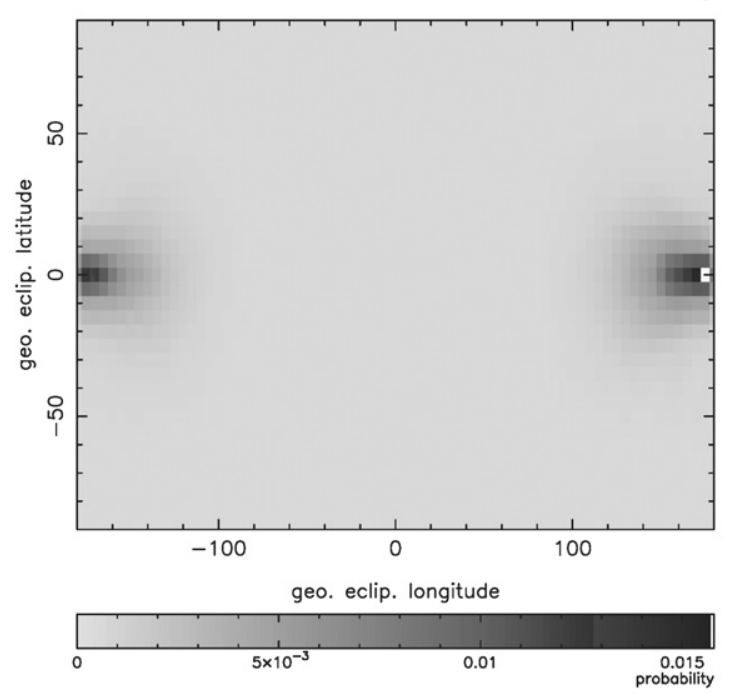

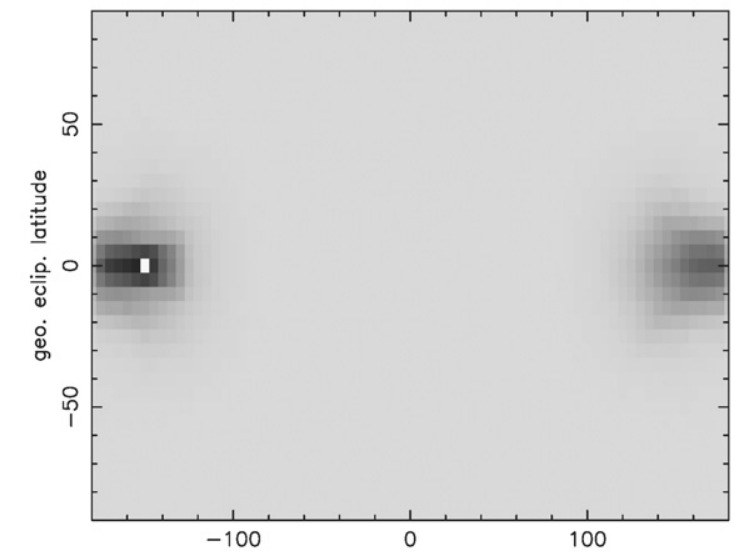

geo. eclip. Iongitude

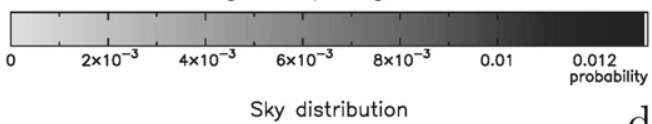

d

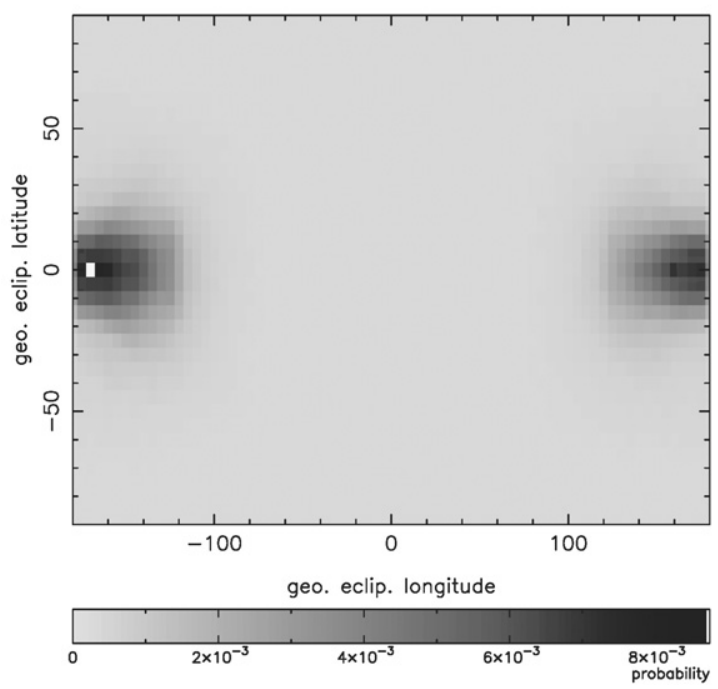

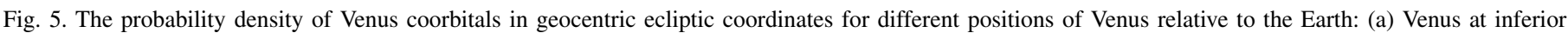

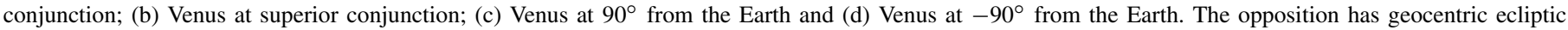
latitude and longitude $0^{\circ}$.

from the Earth are more likely to be concentrated in the direction of the Sun where they cannot be detected. We can quantify the probability densities in different regions of the geocentric coordinate frame. We choose four distinct regions: (I) the observable sky $\left(-100^{\circ}<\right.$ glon $<100^{\circ}$ and $\mid$ glat $\left.\mid<45^{\circ}\right)$; (II) the extended ${ }^{6}$ observable sky $\left(-135^{\circ}<\right.$ glon $<135^{\circ}$ and $\mid$ glat $\left.\mid<45^{\circ}\right)$; (III) the unobservable sky $\left(135^{\circ}<\right.$ glon $<180^{\circ}$ or $-180^{\circ}<$ glon $<-135^{\circ}$ and $\mid$ glat $\mid<45^{\circ}$ ); (IV) the region of high inclination orbits $\left(\mid\right.$ glat $\left.\mid>45^{\circ}\right)$. The probability densities for Venus and Earth coorbitals in these regions are shown in Table 2. The regions I, II and III are represented in Fig. 7.

We see that Venus coorbitals are more likely to be in the unobservable sky (region III) than Earth coorbitals (78\% against $54 \%$ ). Conversely, Venus coorbitals are less likely to be in the

\footnotetext{
6 This includes the region where it is difficult (but not impossible) to detect NEAs due to the proximity of the Sun (see for instance (Tholen et al., 2005)).
}

observable sky (region I) than Earth coorbitals (3\% against $15 \%$ ). Finally, Venus coorbitals are less likely to be on high inclination orbits (region IV) than Earth coorbitals (3.5\% against $9 \%)$.

By comparing Fig. 4b above with Fig. 4b from Paper I we see that the average extinction for Venus coorbitals is typically smaller than the average extinction for Earth coorbitals. In the observable sky this difference in brightness is around -2 while in the extended observable sky this difference drops to around -1 . We conclude that Venus coorbitals are, on average, brighter that Earth coorbitals with the same absolute magnitude.

It is also important to point out that while Venus coorbitals move fast with respect to the Earth (we saw above that the period of relative motion of a coorbital that exactly shares Venus' orbit is 1.6 years), Earth coorbitals move slowly with respect to the Earth (the speed of the motion is dictated by the coorbital 
Table 2

Probability densities for Venus and Earth coorbitals viewed in geocentric frame

\begin{tabular}{|c|c|c|c|c|}
\hline & $\begin{array}{l}\text { Region I } \\
\text { (observable sky) }\end{array}$ & $\begin{array}{l}\text { Region II } \\
\text { (extended observable sky) }\end{array}$ & $\begin{array}{l}\text { Region III } \\
\text { (unobservable sky) }\end{array}$ & $\begin{array}{l}\text { Region IV } \\
\text { (high inclination) }\end{array}$ \\
\hline Venus & $3 \%$ & $18.5 \%$ & $78 \%$ & $3.5 \%$ \\
\hline Earth & $15 \%$ & $37 \%$ & $54 \%$ & $9 \%$ \\
\hline
\end{tabular}

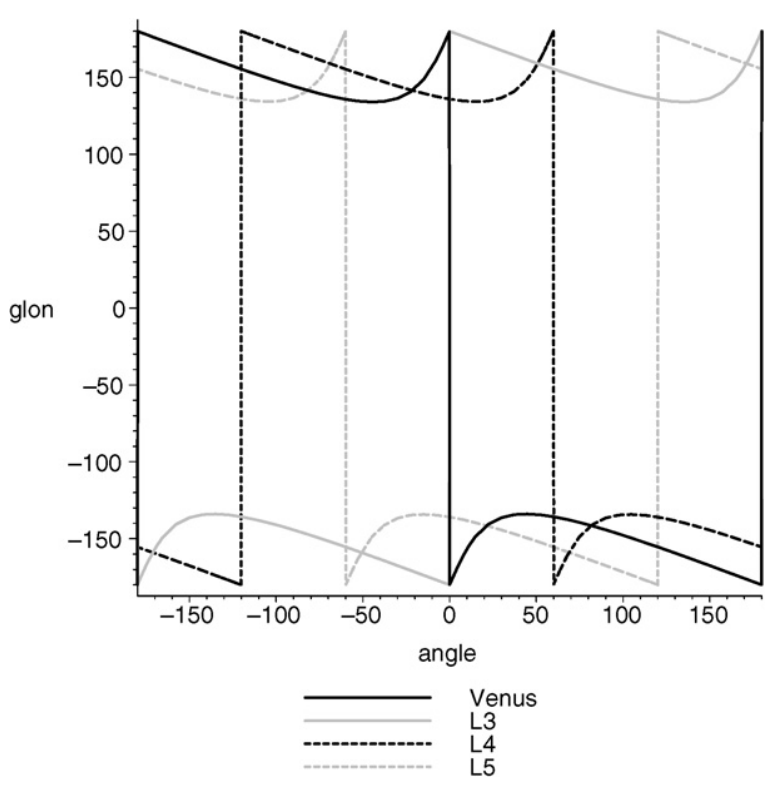

Fig. 6. The geocentric ecliptic longitude of Venus Lagrangian points $L_{3}, L_{4}$ and $L_{5}$ as a function of the position of Venus relative to the Earth represented by the angle Venus-Sun-Earth.

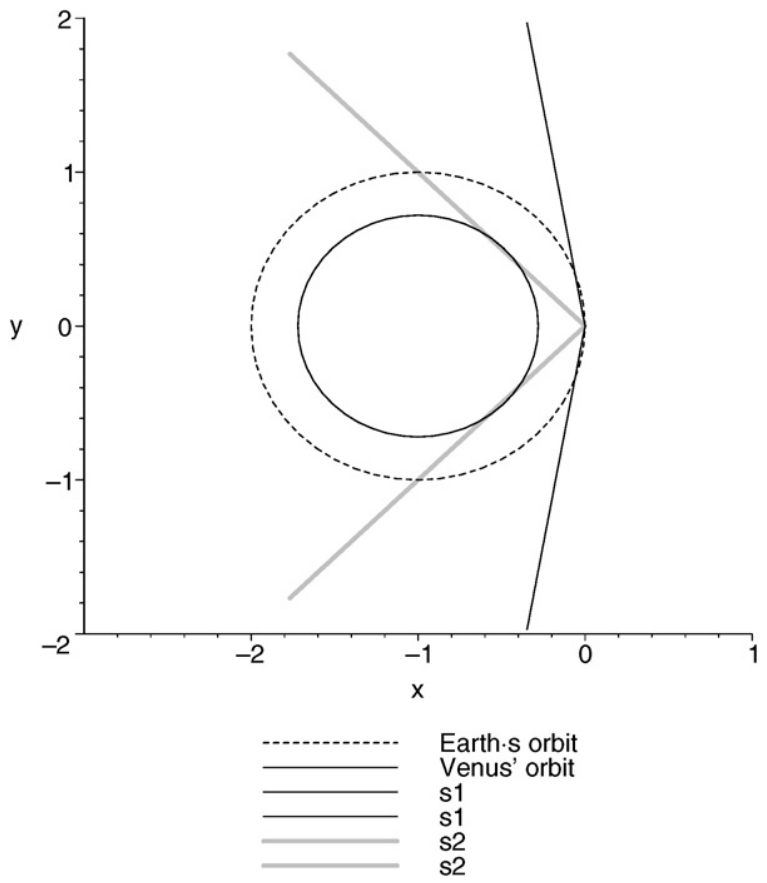

Fig. 7. The ecliptic coordinate frame with the orbits of the Earth (dashed circle) and Venus (solid circle). The gray lines at $\pm 135^{\circ}$ angles separate the extended observable region (to the right) from the unobservable region (to the left). The observable region is limited (on the left) by the black lines at $\pm 100^{\circ}$ angles. period which is about 200 years for Earth tadpole orbits-see footnote 2). Therefore, although Venus coorbitals spend most of the time in the unobservable sky, they can appear frequently, although very briefly, in the observable sky. On the other hand, Earth coorbitals are either in the observable sky at the moment or will probably remain undetected for many decades.

\subsection{Comparison with the sky distribution of trojans}

We also compare our sky distribution of Venus coorbitals with the sky distribution of an hypothetical population of Venus trojans obtained by Evans and Tabachnik (2000). Venus trojans approximately share Venus' orbit at $a=0.72$ AU thus their elongation (the angle Sun-Earth-Object) must be $46^{\circ}>l>$ $-46^{\circ}$. Furthermore, their velocity as seen from Earth has two minima at the extremes of the apparent motion, i.e., at elongation $l= \pm 46^{\circ}$, and a maxima at $l=0^{\circ}$. This explains why the sky distribution of Venus trojans has maxima at $l= \pm 46^{\circ}$, as seen in Fig. 1 of Evans and Tabachnik (2000). In our coordinate frame, $46^{\circ}>l>-46^{\circ}$ corresponds to the regions $-180^{\circ}<$ glon $<-134^{\circ}$ and $134^{\circ}<$ glon $<180^{\circ}$. However, the sky distribution of Venus coorbital NEAs occupies a much broader region. In Fig. $4 \mathrm{a}$ we see that the probability density is still considerable at glon $= \pm 120^{\circ}$ and in Table 2 we see that Venus coorbitals have a small but non-zero (3\%) probability of being in the region with $-100^{\circ}<$ glon $<100^{\circ}$ (this is due to the large amplitude epicyclic motion caused by large eccentricities).

Similarly, we can compare the sky distribution of Earth coorbitals obtained in Paper I with the sky distribution of Earth trojans also obtained by Evans and Tabachnik (2000). The sky distribution of Earth trojans seen in Fig. 2 of Evans and Tabachnik (2000) is, as we would expect, symmetric with respect to $l=0^{\circ}$ and has highest concentration near $L_{4}$ and $L_{5}$ which are located at $l= \pm 60^{\circ}$. As stated in Paper I, the sky distribution of Earth coorbital NEAs does not have maxima near $L_{4}$ or $L_{5}$ which are located at glon $= \pm 120^{\circ}$ in our coordinate frame (Fig. 4a, Paper I). On the other hand, Earth trojans can never be at opposition (in fact, the minimum possible angular separation from the Earth, which is measured along Earth's orbit, is about $6.3^{\circ}$ and occurs at the extrema of the largest amplitude stable horseshoe orbit ${ }^{7}$ ) while Earth coorbital NEAs can (if they are on quasi-satellite orbits or compound orbits that involve quasi-satellite modes like asymmetric horseshoe and asymmetric tadpole orbits-see footnote 1).

\footnotetext{
7 The minimum possible angular separation from the Earth is $(8 \mu) /\left(3 a_{0}\right)$ where $a_{0}=0.74(\mu / 3)^{1 / 3}$ is the normalized half-width of the largest amplitude stable horseshoe orbit (Dermott and Murray, 1981).
} 
The comparisons presented here show that the distributions of Venus and Earth transient coorbitals are very different from distributions of primordial trojans of these planets. However, we would like to point out that these populations of primordial trojans are solely hypothetical as none of these objects was found to date. In fact, the distributions of primordial trojans obtained by Evans and Tabachnik (2000) were based on limited numerical integrations which followed these objects only up to 100 Myr. Recently, Scholl et al. (2005) studied the stability of Venus' trojans performing numerical integrations for the age of the Solar System. They showed that all objects disappear in about 1.2 Gyr hence they conclude that primordial venusian trojans could not have survived to the present date.

\section{Conclusion}

In this paper we obtained the number (according to absolute magnitude $H$ ) and the orbital distribution of NEAs that are expected to be in the 1:1 mean motion resonance with Venus in a steady state scenario. This work is a continuation of a previous article about the population of NEAs that are in the 1:1 mean motion resonance with the Earth (Paper I) and is based on the NEA model developed by Bottke et al. $(2000,2002)$. In short, we followed synthetic NEAs initially located in a region close to the Venusian coorbital region (whose size and orbital distribution we known à priori from the NEA model) and monitored those that are trapped in the 1:1 mean motion resonance with Venus.

We predict that the number of Venus coorbital NEAs with $H<18$ and $H<22$ is $0.14 \pm 0.03$ and $3.5 \pm 0.7$, respectively. The comparison with the current number of Venus coorbitals seems to imply that the survey for these objects is complete up to $H<21$. This result is a little surprising (we do not expect completeness up to such small sizes) and could imply that we are underestimating the population of Venus coorbitals. However, this may also be due to the fact that we obtained average estimates of the number of Venus coorbital NEAs which do not necessarily match the present day observations. In fact, we saw that our results are in good agreement with the work of Christou (2000) who followed the known NEAs in the vicinity of Venus for 200,000 years and calculated the probability of them becoming coorbital with this planet during that timespan.

In Paper I we predicted that the number of Earth coorbital NEAs with $H<18$ and $H<22$ is $0.65 \pm 0.12$ and $16 \pm 3$, respectively. We currently know only one such object, (3753) Cruithne, which lead us to conclude that the survey for Earth coorbitals is probably complete up to $H<18$. These results were also in good agreement with the work of Christou (2000) who followed the known NEAs in the vicinity of the Earth for 200,000 years and calculated the probability of them becoming coorbital with this planet during that timespan.

We obtained the sky distribution of Venus coorbital NEAs and saw that these, as the Earth coorbital NEAs studied in Paper I, spend most of their time away from opposition and are spread over a large sky area. Therefore, these objects are more likely to be found by NEAs search programmes that do not sim- ply observe around opposition and that scan large areas of the sky.

We saw that Venus coorbitals are, on average, brighter than Earth coorbitals with the same absolute magnitude. We also saw that due to the speed of the relative motion with respect to the Earth, Venus coorbitals can move considerably across the sky in a matter of months and thus can reach (and exit) favorable viewing geometries reasonably quickly. On the other hand, Earth coorbitals are either in favorable viewing geometries at the moment or will probably remain undetected for a long time.

Finally, the recent work of Scholl et al. (2005) showed that any primordial population of venusian trojan would have disappeared by now. Therefore, our conclusions regarding the population of transient Venus' coorbitals that we present in this article are the most relevant for any observational searches.

\section{Acknowledgments}

This work was funded by Grant SFRH/BPD/19155/2004 from the "Fundação para a Ciência e Tecnologia" (Portugal) and by Project POCTI/FNU/43656/2001.

\section{References}

Bottke, W.F., Jedicke, R., Morbidelli, A., Petit, J.M., Gladman, B., 2000. Understanding the distribution of Near-Earth Asteroids. Science 288, 2190-2194.

Bottke, W.F., Morbidelli, A., Jedicke, R., Petit, J.M., Levison, H., Michel, P., Metcalfe, T., 2002. De-biased orbital and size distribution of the Near-Earth Objects. Icarus 156, 399-433.

Brasser, R., Innanen, K.A., Connors, M., Veillet, C., Wiegert, P., Mikkola, S., Chodas, P.W., 2004. Transient coorbital asteroids. Icarus 171, 102-109.

Christou, A.A., 2000. A numerical survey of transient coorbitals of the terrestrial planets. Icarus 144, 1-20.

Connors, M., Chodas, P., Mikkola, S., Wiegert, P., Veillet, C., Innanen, K., 2002. Discovery of an asteroid and quasi-satellite in an Earth-like horseshoe orbit. Meteor. Planet. Sci. 37, 1435-1441.

Connors, M., Veillet, C., Brasser, R., Wiegert, P., Chodas, P., Mikkola, S., Innanen, K., 2004. Horseshoe asteroids and quasi-satellites in Earth-like orbits. Lunar Planet. Sci. 35. Abstract 1565.

Dermott, S.F., Murray, C.D., 1981. The dynamics of tadpole and horseshoe orbits. I. Theory. Icarus $48,1-11$

Evans, N.W., Tabachnik, S.A., 2000. Asteroids in the inner Solar System. II. Observable properties. Mon. Not. R. Astron. Soc. 319, 80-94.

Levison, H.F., Duncan, M., 1994. The long-term behavior of short-period comets. Icarus 108, 18-36.

Message, J., 1966. The dominant features of the long-period librations of the Trojan minor planets. In: Barkley Rosser, J. (Ed.), Space Science, Part 2. In: Lectures in Applied Mathematics, vol. 6. American Mathematical Society, Providence, RI, pp. 70-78.

Michel, P., 1997. Overlapping of secular resonances in a Venus horseshoe orbit. Astron. Astrophys. 328, L5-L8.

Mikkola, S., Brasser, R., Wiegert, P., Innanen, K., 2004. Asteroid 2002 VE68: A quasi-satellite of Venus. Mon. Not. R. Astron. Soc. 351, L63-L65.

Morais, M.H.M., Morbidelli, A., 2002. The population of Near-Earth Asteroids in coorbital motion with the Earth. Icarus 160, 1-9.

Morbidelli, A., Bottke, W., Michel, P., Froeschlé, Ch., 2002. Origin and evolution of Near Earth Asteroids. In: Bottke, W., Paolicchi, P., Binzel, R.P., Cellino, A. (Eds.), Asteroids III. Univ. of Arizona Press, Tucson, pp. 409422 .

Namouni, F., 1999. Secular interactions of coorbiting objects. Icarus 137, $293-$ 314. 
Nesvorný, D., Thomas, F., Ferraz-Mello, S., Morbidelli, A., 2002. A perturbative treatment of the coorbital motion. Celest. Mech. Dynam. Astron. 82, 323-361.

Scholl, H., Marzari, F., Tricarico, P., 2005. The instability of Venus trojans. Astron. J. 130, 2912-2915.

Tholen, D., Bernardi, F., Tucker, R.A., 2005. Probing the depths of the Solar System: A tale of two asteroids. In: DPS Meeting 37, American Astronomical Society. Abstract 3.06.
Wiegert, P.A., Innanen, K.A., 1997. An asteroidal companion to the Earth. Nature $387,685-686$.

Wiegert, P., Connors, M., Chodas, P., Veillet, C., Mikkola, S., Innanen, K., 2002. Earth coorbital objects. Eos. Trans. AGU. 83 (47). Abstract P11A0352 .

Wisdom, J., Holman, M., 1991. Symplectic maps for the N-body problem. Astron. J. 102, 1528-1538. 